\title{
Acylation of 1-[2-(2-Methoxyethoxy)ethyl]pinacolone: Preparation of 4-[2-(2-Methoxyethoxy)ethyl]-2,2,6,6-tetramethylheptane-3,5-dione
}

\author{
Seung Woo Woo, Hyoung Cheul Kim, Young Woo Oh, In Kwon Youn, ${ }^{\dagger}$ and Hyoung Rae Kim \\ Bioorganic Science Diwision, Korea Research Institute of Chemical Technologw, P.O. Box 107, Yusong. Taejon 305-600, Korea \\ ${ }^{\dagger}$ Department of Chemistry, Paichai Liniversity, 439-6 Doma-dong, Su-Ku, Taejon 302-735, Korea \\ Received August 31, 2001
}

Keywords : TMHD. CVD. Ligand. $\beta$-Diketone, $\alpha$-Acylation.

Substituted or non-substituted 2,2,6.6-tetramethy lheptane3,5-dione (TMHD) has been used as a ligand of a precursor to the chemical vaporization of deposition (CVD) ${ }^{1,2} \alpha$ Alkyation of $\beta$-diketones is generally a very slow reaction. affording low yields due to serious competition from $O$ alkylation. ${ }^{3,5}$ When activated electrophiles. such as allyl bromide, propargyl bromide. or benzyl bromide, ${ }^{+5}$ react with acety lacetone, the yield of $C$-allylation is exceptionally good: however. reactions of highly substituted $\beta$-diketone with long chained electrophiles show very low yields of $C$ alkylated products. ${ }^{6}$ Moreover, alkylation of TMHD itself has no examples. We examined the direct alkylation of TMHD with 2-(2-methoxyethoxy)ethyl iodide under various conditions, and we found no reaction occurred at all even after 3 days heating in THF or DMF with bases such as sodium hydride. sodium carbonate, potassium carbonate, or cesium carbonate. ${ }^{7}$ To synthesize 4 -[2-(2-methoxyethoxy)ethyl]-2,2.6,6-tetramethylheptane-3.5-dione (4) we should detour $^{8,9}$ as shown in Scheme 1. Pinacolone (1) was monoalkylated by 2-(2-methoxyethoxy)ethyl iodide (2) with lithiun diisopropylanide (LDA) as a base to form [2-(2methoxyethosy)ethyl]-2.2-dimethylbutan-3-one (3) in good yield. To acylate $\dot{3}^{110}$ by trimethylacetyl chloride. we first used LDA as a base at $-78^{\circ} \mathrm{C}$ in THF. After the reaction mixture was stirred at $-78^{\circ} \mathrm{C}$ for $2 \mathrm{~h}$, we isolated twice the amount of $O$-acylated product $5^{11}$ compared with $\mathrm{C}$-acylated product $4^{12}$ in a total yield $69 \%$ (Entry 1 in Table 1 ). We examined lithium bis(trimethylsilyl)anide (lithiun HMDS), sodium bis(trimethy lsilyl)anide (sodium HMDS), potassium bis(trimethylsilyl)anide (potassium HMDS), and sodium

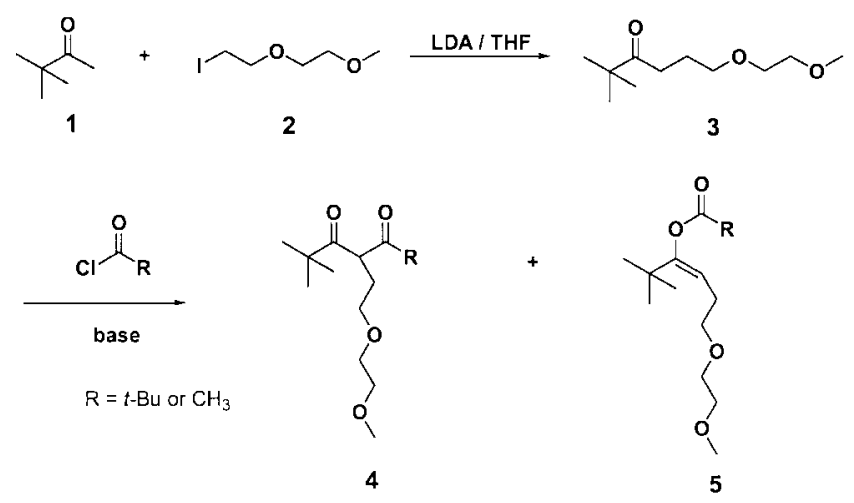

Scheme 1 hydride as bases under the same reaction conditions (Table 1). $O$-Acylations of $\mathbf{3}$ were favored slightly in this acylation, but when potassium HMDS was used as a base. (-acylation was favored to a small extent (Entry 4 and 8 in Table 1). In the case of sodium hydride. almost no reaction occurred even at $\mathrm{rt}$ (Entry $5 \mathrm{in}$ Table 1). The addition of cesium carbonate or substitution of solvent to toluene showed no significant effects in the percentage of $O$-acylation and $C$ acylation. We examined the acylation of 3 with acetyl chloride instead of trimethylacetyl chloride to investigate the effect of the bulkiness of electrophiles. and we found $C$ alkylation was more favored as expected (Entry 10, 11. and 12 in Table 1). When potassium HMDS was used as a base in toluene. the best result was obtained in acetylation and also good result in trimethylacetylation. But, we prefer THF due to the easy removal of solvent in the purification process. Next. we changed the electrophile from trimethy lacetyl clloride to the corresponding cyanide. ${ }^{13.14}$ anhydride. or ester to change the softness of the electropliles. Under the same reaction conditions, we applied a different set of electrophiles in THF, and the results are summarized in Scheme 2 and Table 2. When trimethylacetyl cyanide was used as an electrophile, significantly improved results were obtained (Entry 1 and 2 in Table 2). "W When potassium

Table 1. Acylation of 4-[2-(2-Methosyethoxy )ethyl]-2,2-dimethy]butan-3-one (3) by Trimethylacetyl Chloride or Acetyl Chloride with Various Bases

\begin{tabular}{|c|c|c|c|c|c|}
\hline Entry & $\mathrm{R}$ & Base & $\begin{array}{l}\text { Reaction tempera- } \\
\text { ture/Solvent }\end{array}$ & $\begin{array}{c}+ \\
(\%)^{a}\end{array}$ & $\begin{array}{c}5 \\
(\%)^{2}\end{array}$ \\
\hline 1 & $t-\mathrm{Bul}$ & LDA & $-78^{\circ} \mathrm{C} / \mathrm{THF}$ & 24 & 45 \\
\hline 2 & $t-\mathrm{Bu}$ & Lithium HMDS & $-78^{\circ} \mathrm{C} / \mathrm{THF}$ & 30 & 39 \\
\hline 3 & $t-\mathrm{Bul}$ & Sodium HMDS & $-78^{\circ} \mathrm{C} / \mathrm{THF}$ & $<10$ & $<10$ \\
\hline 4 & $t-\mathrm{Bul}$ & Potassium HMDS & $-78^{\circ} \mathrm{C} / \mathrm{THF}$ & 42 & 36 \\
\hline 5 & $t-\mathrm{Bul}$ & Sodium hydride & $24^{\circ} \mathrm{C} / \mathrm{THF}$ & trace & trace \\
\hline 7 & $t-\mathrm{Bu}$ & Lithium HMDS & $-78^{\circ} \mathrm{C} /$ Tolune & 26 & 67 \\
\hline 8 & $t-\mathrm{Bul}$ & Potassium HMDS & $-78^{\circ} \mathrm{C} /$ Toluene & 35 & 27 \\
\hline 9 & $t-\mathrm{Bu}$ & $\begin{array}{l}\text { Lithium HMDS/ } \\
\text { cesium carbonate }\end{array}$ & $-78^{\circ} \mathrm{C} /$ Toluene & 26 & 20 \\
\hline 10 & $\mathrm{CH}_{3}$ & Lithium HMDS & $-78^{\circ} \mathrm{C} / \mathrm{THF}$ & 32 & 31 \\
\hline 11 & $\mathrm{CH}_{3}$ & Potassium HMDS & $-78^{\circ} \mathrm{C} / \mathrm{THF}$ & 51 & 30 \\
\hline 12 & $\mathrm{CH}_{3}$ & Potassium HMDS & $-788^{\circ} \mathrm{C} /$ Toluene & 53 & 14 \\
\hline
\end{tabular}

Isolated vields 


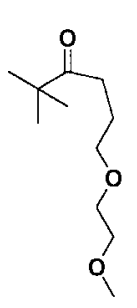

3
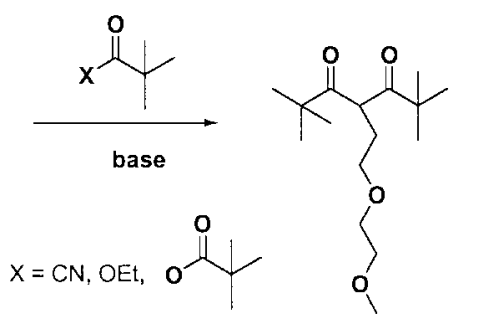

4

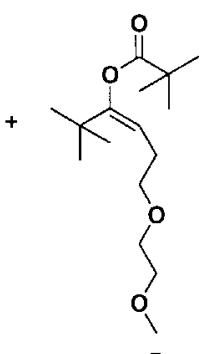

5
Scheme 2

Table 2. Acylation of 4-[2-(2-Methoxyethoxy)ethyl]-2,2-dimethylbutan-3-one (3) by Various Electrophiles

\begin{tabular}{|c|c|c|c|c|c|}
\hline Entry & $\mathrm{X}$ & Base & $\begin{array}{l}\text { Reaction Tem- } \\
\text { perature/Solvent }\end{array}$ & $\begin{array}{c}4 \\
(\%)^{a}\end{array}$ & $\begin{array}{c}5 \\
(\%)^{a}\end{array}$ \\
\hline 1 & $\mathrm{CN}$ & Lithium HMDS & $-78^{\circ} \mathrm{C} / \mathrm{THF}$ & 74 & 17 \\
\hline 2 & $\mathrm{CN}$ & $\begin{array}{l}\text { Potassium } \\
\text { HMDS }\end{array}$ & $-78^{\circ} \mathrm{C} / \mathrm{THF}$ & 80 & 9 \\
\hline 3 & OCOBll-t & Lithium HMDS & $-78^{\circ} \mathrm{C} / \mathrm{THF}$ & 54 & 6 \\
\hline 4 & $\mathrm{OCH}_{2} \mathrm{CH}_{3}$ & Lithium HMDS & $-78^{\circ} \mathrm{C} / \mathrm{THF}$ & trace & trace \\
\hline
\end{tabular}

"Isolated yields

HMDS was used as a base. the yield of C-acylation increased to $80 \%$ and the ratio of $C$-acylation to 3 was much improved to $9: 1$ in total $89 \%$ isolated yield (Entry 2 in Table 2). Even when lithium HMDS was used as a base. C. alkylation was much more favored (Entry 1 in Table 2) than with trimethy lacetyl chloride (Entry 2 in Table 1). Reaction with anlyydride also showed improved results compared with acid chloride (Entry 3 Table 2), whereas ester did not react at all (Entry 4 in Table 2).

4-[2-(2-Methoxyethoxy)ethyl]-2.2,6,6-tetramethylheptane3,5-dione $(4)$, which is expected to be useful as a ligand of a precursor in CVD was synthesized from 4-[2-(2-methosyethoxy)ethyl]-2.2-dimethylbutan-3-one (3) and trimethylacetyl cyanide with potassium HMDS as a base in good yield. We will synthesize other derivatives by this procedure and test the resulting compounds as ligands of precursors.

\section{References}

1. Kirsty. A. F: O'Brien, P.: Otway, D. I: White. A. J. P. Williams,
D. J.; Jones, A. C. Horg. Chem. 1999, 38. 1432.

2. Beachley. O. I.. Ir.: Tames. R. G.: Churchill. M. R. Organontetallics 2000. 19. 4544 .

3. Kurts. A. L.: Getnkina. N. K.: Maclas. A.: Beletskaya. I. P.: Reutov, O. A. Tetrohedron 1971,27,4777

4. Lloris. M. E.; Galvez. N.: Marquet. J.: Moreno-Manas, M. Tetrahedron 1991, 47.8031.

5. Ranu. B. C.: Bhar. S. J. Chent Soc. Perkin Trans. I 1992. 3. 365.

6. Zanina. A. S.: Shergina. S. I.: Sokolor. I. E.: Shwartsberg. M. S. Izt. Akad Nauk, Ser Khint 1996. 10.2518.

7. Shrout. D. P.: Lightner. D. A. Symth. Conmun. 1990, $20,2075$.

8. Tirpak. R. E.: Rathke, M. W. J. Org. Chem 1982, 47, 5099.

9. (3): 'H NMR $\left(200 \mathrm{MHz} . \mathrm{CDCl}_{3}\right) \delta 1.03-1.07$ (m. 9H). 1.75-1.81 (m. $2 \mathrm{H}) .2 .21-2.60(\mathrm{~m} .2 \mathrm{H}) .3 .31$ (s. $3 \mathrm{H}) .3 .36-3.48(\mathrm{~m} .6 \mathrm{H}):$ IR $\left(\mathrm{cm}^{-1}\right)$ 1107. 1479. 1705.2873.2966.

10. (5. $\mathbf{R}=$ t-butyl): 'H NMR (200 MHz. $\left.\mathrm{CDCl}_{3}\right) \delta 1.05-1.32(\mathrm{~m}$. $18 \mathrm{H}) .2 .08(\mathrm{~g} J=14.04 \mathrm{~Hz}, 2 \mathrm{H}) .3 .38-3.57(\mathrm{~m} .9 \mathrm{H}), 5.12(\mathrm{s.} \mathrm{lH}):$ ${ }^{13} \mathrm{C}$ NMR $\delta 26.35,27.32,27.92 .26 .18 .39 .15,59.00,69.83,70.19$. $71.85,109.13 .155 .78,175.59: \mathrm{IR}\left(\mathrm{cm}^{-1}\right) 1122.1747,2873,2972$ HRMS calcd for $\mathrm{C}_{16} \mathrm{H}_{31} \mathrm{O}_{4}\left(\mathrm{MH}^{+}\right)$287.2218. found 287.2222. (5. $\mathbf{R}=$ methyl): ${ }^{1} \mathrm{H}$ NMR (200 MHz. CDCl 3$) \delta 1.03-1.13$ (m. 9H). $2.07-2.16(\mathrm{~m} .5 \mathrm{H}) .3 .33-3.57$ (m. $9 \mathrm{H}) .5 .12(\mathrm{t} . J=14.04 \mathrm{~Hz} .1 \mathrm{H})$ : ${ }^{13}$ C NMR $\delta 20.61,26.39,26.71 .27 .93,30.91,36.02,59.05,69.92$. $70.16,71.87,109.36 .156 .03 .168 .43:$ IR $\left(\mathrm{cm}^{-1}\right) 1207.1758,2872$. 2969: HRMS calcd for $\mathrm{C}_{13} \mathrm{H}_{25} \mathrm{O}_{4}\left(\mathrm{MH}^{-}\right) 245.1757$, found 245.1753.

11. (4. $\mathbf{R}=$-butyl): ${ }^{1} \mathrm{H}$ NMR (200 MHz. $\left.\mathrm{CDCl}_{3}\right) \delta 1.11-1.29$ (m. I8H). 2.07 (q. $J=6.51 \mathrm{~Hz} .2 \mathrm{H}) .3 .28-3.35(\mathrm{~m} .5 \mathrm{H}) .3 .48(\mathrm{t} . J=$ $7.52 \mathrm{~Hz}, 4 \mathrm{H}), 4.71(\mathrm{t}, J=13.02 \mathrm{~Hz}, \mathrm{lH}):{ }^{13} \mathrm{C}$ NMR 27.26. 29.41 , $30.91,41.57 .51 .76 .58 .99,68.94,70.09 .71 .84,210.93 ; \mathrm{IR}\left(\mathrm{cm}^{-1}\right)$ $1105,1691.1719,2874.2966$ : HRMS calcd for $\mathrm{C}_{14} \mathrm{H}_{31} \mathrm{O}_{4}\left(\mathrm{MH}^{+}\right)$ 287.2223. found 287.2222. (4. R = methyl) ' $\mathrm{H}$ NMR (200 MHz. $\mathrm{CDCl}_{3}$ ) $\delta 1.14$ (s. $9 \mathrm{H}$ ). 2.17 (s. $3 \mathrm{H}$ ). 3.36 (s. $3 \mathrm{H}$ ). $3.42-3.62$ (m. $8 \mathrm{H}) .4 .38(\mathrm{t} . j=8.90 \mathrm{~Hz} . \mathrm{IH}) .{ }^{13} \mathrm{C}$ NMR $\delta 26.40 .27 .27 .29 .42$. $44.59,51.78 .59 .01 .68 .96,69.87,70.10 .71 .82,210.93 ; \mathrm{IR}\left(\mathrm{cm}^{-1}\right)$ $1105,1479,1720.2873,2964$.

12. Tang. Q.: Sen, S. E. Tetohledron Lett. 1998, 39. 2249.

13. Sperber. N.: Fricano. R. J. Am. Chem. Soc. 1950. 72. 2792.

14. Kraus. G. A.: Dneprovskaia. E. Tetrohedron Letf 2000. H. 21.

15. Reaction of [2-(2-methoxyethoxy) ethyl]-2.2-dimethylbutan-3-one (3) with trimethylacetyl cyanide. To a solution of [2-(2-methoxyethoxy)ethyl]-2.2-dimethylbutan-3-one (3) $(0.50 \mathrm{~g}, 2.47 \mathrm{mmol})$ in anhydrous THF $(25 \mathrm{~mL})$ at $-78^{\circ} \mathrm{C}$ was added potassium bis (trimethylsilyl amide $(0.5 \mathrm{M}$ solution in toluene. Aldrich. $7.41 \mathrm{~mL})$ and the reaction misture was stirred for $2 \mathrm{~h}$. And then. trimethylacetyl cyanide $(0.4 \mathrm{l} \mathrm{g} .3 .7 \mathrm{lmmol})$ was dropwisely added by a syringe. After being stirred for $2 \mathrm{~h}$. the reaction mixture was poured into ice-water and extracted with ethyl acetate. The organic laver was dried over magnesium sulfate, concentrated under reduced pressure. and the residue was purified by chromatography on silica gel (EtOAc Hexane. 411$)$ to give (4) $(0.57 \mathrm{~g} .80 \%$ ) and (5) $(0.06 \mathrm{~g}$. $9^{\circ}$ ) as a pale yellow oil. 